\title{
Comparative Study of Dwarf Mutants of Maize (Zea mays L.)
}

Victoria C. Cheng ${ }^{1}$, Wayne Y. Cheng ${ }^{2}$, Ping-chin Cheng ${ }^{3}$ and David B. Walden ${ }^{4}$

${ }^{1}$ Williamsville East High School, Williamsville, NY 14051 USA

${ }^{2}$ Dept. of Biological Sciences, Cornell University, Ithaca, NY 14853 USA

${ }^{3}$ Dept. of Electrical Engineering, State Univ. of New York, Buffalo, NY 14260 USA

${ }^{4}$ Dept. of Biology, University of Western Ontario, London, Canada N6A 5B7

There are number of maize dwarf mutants which are characterized by their stubby appearances. In our previous study, we reported that both $n a l$ and $n a 2$ mutants develop unusual node and internodes. The nal/nal homozygous develops no internode in the vegetative growth portion of the stem, resulting in the entire stem to become structurally resemblance of a node; in contrast, the $n a 2 / n a 2$ develops a slanted node, which continue from node to node making all the nodes are essentially connected ${ }^{[1,2,3]}$. In addition to the $n a l$ and $n a 2$ dwarf mutants, we have conducted a study on an additional nembers of dwarf mutants, namely, the $d 1, d 5$ and $D 8$. The $d 1$ and $d 5$ are recessive and $D 8$ is dominant.

Maize plants were grown at the experimental station of the Univ. of Western Ontario in the summer of 2003 and 2004. Figure 1-3 shows the phenotype of $d 1 / d 1, d 5 / d 5$ and $D 8 /+$. The $d 5 / d 5$ individual shown also expresses the $y s 3 / y s 3$ as the plant shows striated leave. FIG 1-3 shows all the mutants have relatively short leaf blades but appear to have similar blade width as the wild type. Plants were collected at/after silking of the upper ear, fixed in methanol and cut open longitudinally. After cutting open, the specimen was soaked in isopropyl alcohol to remove the brown coloration. Specimens were then recorded by using a modified flat-bed scanner ${ }^{[4]}$. In order to visualize the vascular architecture under optical microscope, fixed specimen were washed in water and digested by enzymatic treatment as descried by Cheng et. al. ${ }^{[5]}$. FIG 4 shows longitudinal sections of the mutants; all three mutants have normal node-internodes architecture but develop significantly shorter and stubby internodes. This short internodes development also appears in the tiller (t) and ear (e) branch. The $d l / d l$ shown in FIG 4 also expresses $r t 1 / r t 1$ (rootless 1) as it has very poor root development. Close examine the structure of dwarf mutants reveal normal node architecture similar to the wild type. FIG 5 and 6 are optical micrographs showing the architecture of vasculature in the node region of a wild-type (Ohio 43/KYS) plant. Branching of vasculature occurs only at the node which forms complex nodal network supplying leaf and branch.

[1] W. Y. Cheng et al. Maize Genet. Coop. News Lett. (2001) Vol. 75, 6-8.

[2] P. C. Cheng et al. Scanning (2001) 23, 98-99.

[3] W. Y. Cheng et al. Scanning (2001) 23, 136-137.

[4] W. Y. Cheng et al. Scanning (2001) 23, 135-136.

[5] W. Y. Cheng et al. Maize Genet. Coop. News Lett. (2002) Vol. 76, 29. 


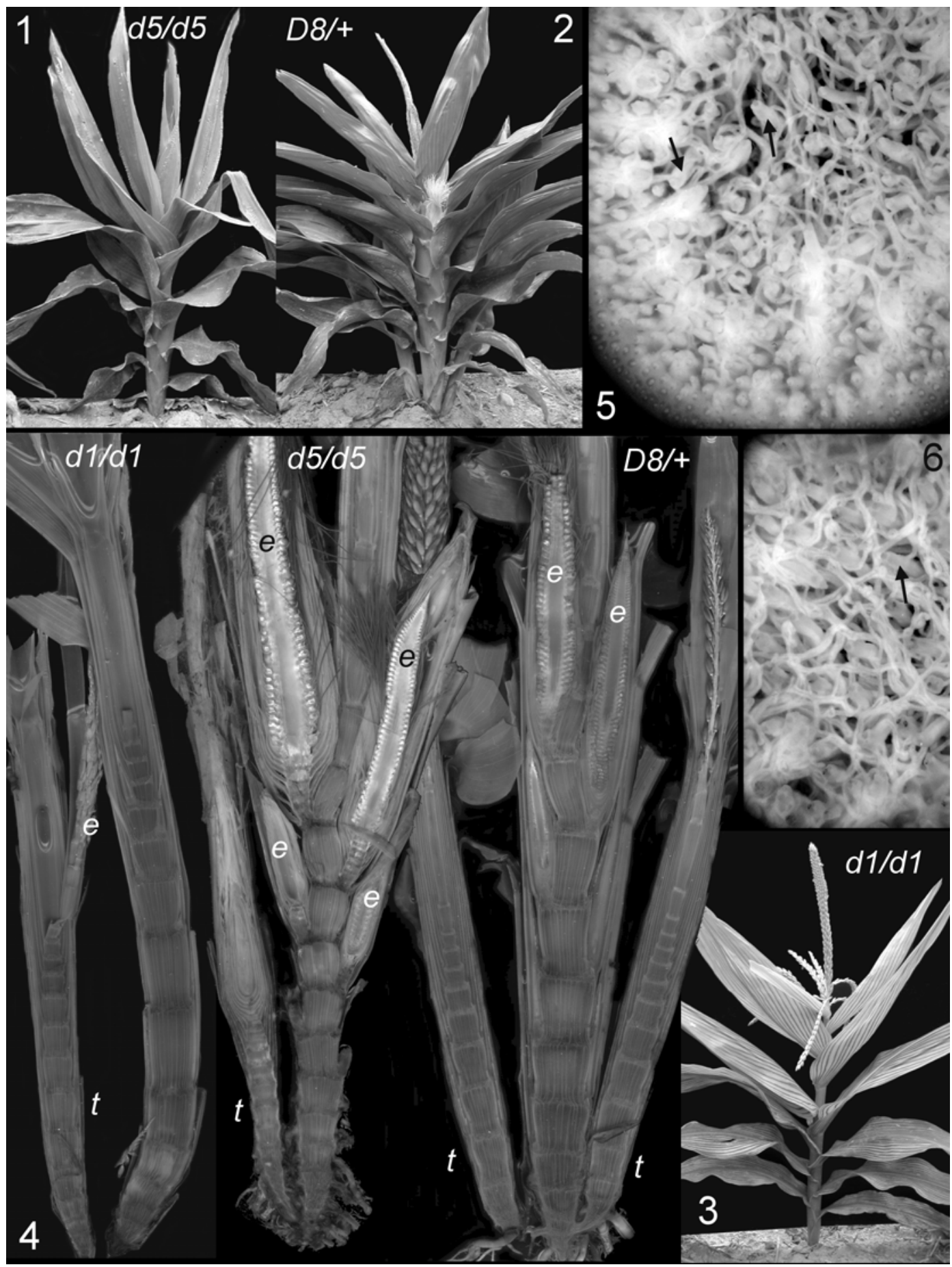

FIG. 1, 2 and 3. Phenotype of $d 1 / d 1$ (also express $y s 3 / y s 3$ ), $d 5 / d 5$ and $D 8 /+$

FIG. 4. Latitudinal section of stem of $d 1 / d 1$ (also express $r t 1 / r t 1$ as poor root development is evident), $d 5 / d 5$ and $D 8 /+$. t: tiller, e: ear.

FIG. 5 and 6. Micrographs showing enzyme digested node region in the stem of wild-type $(+/+)($ Ohio $43 /$ KYS $)$. Arrows show vascular bundles running through the node. Note the branching of vascular bundles which forms the nodal network. 